\title{
Erosive Nipple Adenomatosis
}

National Cancer Institute

\section{Source}

National Cancer Institute. Erosive Nipple Adenomatosis. NCI Thesaurus. Code C4383.

A rare benign neoplasm that arises in the area of the nipple. Clinically, it usually presents as a tender erythematous crusting lesion with hardening of the nipple. Morphologically, there is proliferation of ducts lined with epithelial and myoepithelial cells and focal erosion of the epidermis. 